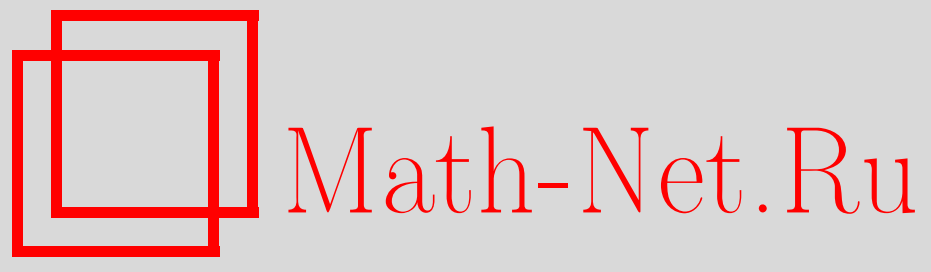

Е. И. Тимошенко, Два замечания о матрицах над групповыми кольцами, Матем. заметки, 2016, том 99, выпуск $1,155-157$

DOI: https://doi.org/10.4213/mzm10595

Использование Общероссийского математического портала Math-Net.Ru подразумевает, что вы прочитали и согласны с пользовательским соглашением http://www . mathnet.ru/rus/agreement

Параметры загрузки:

IP : 34.229 .108 .108

26 апреля 2023 г., 09:21:06

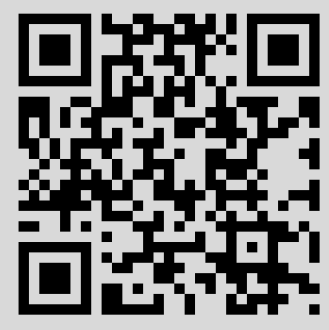




\section{Два замечания о матрицах над групповыми кольцами}

\section{Е. И. Тимошенко}

Хорошо известны примеры ассоциативных колец с единицей, в которых некоторые необратимые элементы имеют правые либо левые обратные. Для групповых колец ситуация иная. Пусть $\mathbb{C}$ - поле комплексных чисел, $G$ - произвольная группа, $\mathbb{C}[G]-$ групповое кольцо группы $G$ над полем $\mathbb{C}$. Капланский [1] доказал, что если некоторый элемент $\alpha \in$ $\mathbb{C}[G]$ имеет левый обратный, то он обратим. Простое алгебраическое доказательство этой теоремы нашла Монтгомери [2]. Теорема Капланского справедлива, если поле $\mathbb{C}$ заменить любым полем характеристики ноль [3; с. 38]. Отметим, что для полей простой характеристики вопрос открыт.

Известно [1], [2], что каждая матрица порядка $n$ с элементами из алгебры $\mathbb{C}[G]$, имеющая правую или левую обратную, обратима. Это свойство полезно при исследовании групп и их групповых колец. Таким образом, кольца матриц над групповыми кольцами обладают дополнительными "хорошими" свойствами по сравнению с кольцами матриц над произвольными ассоциативными кольцами. Возникает вопрос насколько широко верны подобные "хорошие" свойства. Например, совпадают ли для групповых колец понятия унимодулярных элементов, определенных с помощью левых и правых идеалов.

Напомним соответствующие определения. Пусть $R$ - ассоциативное кольцо с единицей, $n$ - положительное целое число, $R^{n}$ - свободный левый $R$-модуль ранга $n$. Согласно определению из книги [4] элемент $\mathbf{r}=\left(r_{1}, \ldots, r_{n}\right) \in R^{n}$ называется унимодулярным, если левый идеал, порожденный элементами $r_{1}, \ldots, r_{n}$, совпадает с кольцом $R$. Другими словами

$$
\left(r_{1}^{\prime}, \ldots, r_{n}^{\prime}\right)\left(r_{1}, \ldots, r_{n}\right)^{T}=1
$$

для некоторых $r_{i}^{\prime} \in R, i=1, \ldots, n$. Обозначим так определенное множество унимодулярных (слева) элементов через $U m_{l}^{n}(R)$.

Естественно определить множество $U m_{r}^{n}(R)$ как множество тех элементов из $R^{n}$, для которых правый идеал кольца $R$, порожденный элементами $r_{i}, i=1, \ldots, n$, совпадает с $R$, т.е.

$$
\left(r_{1}, \ldots, r_{n}\right)\left(r_{1}^{\prime \prime}, \ldots, r_{n}^{\prime \prime}\right)^{T}=1
$$

для некоторых $r_{i}^{\prime \prime} \in R, i=1, \ldots, n$. Именно так определены, например, унимодулярные элементы в обзоре [5] Артамонова и Бовди.

Возникает вопрос, совпадают ли множества $U m_{l}^{n}(R)$ и $U m_{r}^{n}(R)$, если в качестве $R$ рассматривать групповые кольца $\mathbb{C}[G]$, например, над полем комплексных чисел. Из теоремы Капланского получаем $U m_{l}^{n}(\mathbb{C}[G])=U m_{r}^{n}(\mathbb{C}[G])$ при $n=1$. Это позволяет предположить утвердительный ответ при $n \geqslant 2$. Однако, как мы покажем, для широкого класса групповых колец $R=\mathbb{C}[G]$ равенство $U m_{l}^{n}(R)=U m_{r}^{n}(R)$ несправедливо при $n \geqslant 2$.

Группа $G$ называется unique-product group (сокращенно $U P$-груnпа), если для любых двух непустых подмножеств $X$ и $Y$ из $G$ существует элемент $g \in G$, имеющий единственную запись вида $g=x y, x \in X, y \in Y$.

$U P$-группами являются, например, все правоупорядоченные группы, а также локально индикабельные группы.

Если $G$ является $U P$-группой, а $P$ - поле характеристики ноль, то групповая алгебра $P[G]$ имеет только тривиальные единицы, т.е. элемент $h \in P[G]$ обратим тогда и только тогда, когда $h=\lambda g$ для некоторого $\lambda \in P \backslash\{0\}, g \in G$ (см., например, [3], [6]).

Работа выполнена при поддержке Российского фонда фундаментальных исследований (грант № 15-01-485), а также при финансовой поддержке Министерства образования и науки (государственное задание № 2014/138, проект 1052).

DOI: $10.4213 / \mathrm{mzm} 10595$ 
Будет указан еще один пример нарушения "хороших" свойств матрицы. А именно, попутно с доказательством несовпадения множеств $U m_{l}^{n}(R)$ и $U m_{r}^{n}(R), n \geqslant 2$, для любого поля $P, \operatorname{char} P \neq 2$, и любой неабелевой $U P$-группы $G$ будет указана обратимая матрица $M$ над алгеброй $R=P[G]$ такая, что транспонированная матрица $M^{T}$ необратима.

Tеорема 1. Пусть $G$ - неабелева UP-группа и $P$ - поле характеристики 0 . Тогда найдется обратимая матрица $M$ над алгеброй $P[G]$ такая, что транспонированная матрииа $M^{T}$ необратима.

ДоказАтельство. Пусть элементы $g$ и $h$ из $G$ не перестановочны. Тогда элемент $1+$ $g h-h g$ необратим в кольце $P[G]$.

Рассмотрим матрицы

$$
A=\left(\begin{array}{ll}
1 & g \\
0 & 1
\end{array}\right), \quad B=\left(\begin{array}{ll}
1 & 0 \\
h & 1
\end{array}\right) .
$$

Их произведение

$$
M=A B=\left(\begin{array}{cc}
1+g h & g \\
h & 1
\end{array}\right)
$$

является обратимой матрицей, так как обратимы $A, B$. Докажем, что транспонированная матрица $M^{T}$ необратима. Пусть матрица

$$
C=\left(\begin{array}{ll}
a & b \\
c & d
\end{array}\right)
$$

обратная к $M^{T}$. Имеем

$$
\left(\begin{array}{cc}
1+g h & h \\
g & 1
\end{array}\right)\left(\begin{array}{ll}
a & b \\
c & d
\end{array}\right)=E
$$

Значит,

$$
\begin{aligned}
(1+g h) a+h c & =1, \\
g a+c & =0 .
\end{aligned}
$$

Из (2) имеем

$$
c=-g a .
$$

Из (1) получим

$$
(1+g h) a+h(-g a)=1 .
$$

Значит, элемент $1+g h-h g$ обратим. Противоречие доказывает теорему.

Теорема 2. Пусть $N$-свободная 2-ступенно нильпотентная группа ранга 2 с базисом $\{x, y\}, G$ - произвольная группа, для которой существует гомоморфизм на группу $N$, $P$ - произвольное поле, характеристика которого не равна 2 . Тогда при $n \geqslant 2$ множества элементов $U m_{l}^{n}(P[G])$ и $U m_{r}^{n}(P[G])$ различны.

Доказательство. Пусть $g$ и $h$ прообразы элементов $x$ и $y$, соответственно, при указанном в теореме гомоморфизме. Рассмотрим матрицы $A$ и $B$, определенные в теореме 1 . Тогда

$$
D=A B A=\left(\begin{array}{cc}
1+g h & 2 g+g h g \\
h & 1+h g
\end{array}\right) .
$$

Матрица $D$ обратима. Поэтому элемент $(1+g h, 2 g+g h g)$ принадлежит $U m_{r}^{2}(P[G])$. Предположим, что этот элемент принадлежит $U m_{l}^{2}(P[G])$. Тогда элемент $(1+x y, 2 x+x y x)$ принадлежит $U m_{l}^{2}(P[N])$. Значит, для некоторых $u_{1}, v_{1} \in P[N]$ имеем

$$
u_{1}(1+x y)+v_{1}(2+y x)=1 .
$$

Пусть $y x=x y c$. Элемент $c$ порождает центр группы $N$. Получим

$$
u_{2}(1+x y)+v_{2}(2-c)=1
$$


для некоторых $u_{2}, v_{2} \in P[N]$. Элементы $\{x y, y\}$ образуют базис группы $N$. Поэтому

$$
u(1+x)+v(2-c)=1, \quad u, v \in P[N] .
$$

Любой элемент $\gamma \in P[N]$ можно однозначно представить в виде

$$
\gamma=\sum_{i} y^{i} g_{i}(x, c)
$$

где $i$ пробегает некоторое конечное множество целых значений, а $g_{i}(x, c)$ - многочлены Лорана от переменных $x$ и $c$. Напомним, что многочлены Лорана содержат целые, в том числе и отрицательные, степени переменных $x$ и $c$. Пусть

$$
u=\sum_{i} y^{i} g_{i}(x, c), \quad v=\sum_{j} y^{j} f_{j}(x, c) .
$$

Тогда из (3) получаем

$$
g_{0}(x, c)(1+x)+f_{0}(x, c)(2-c)=1 .
$$

Применим к равенству (4) эндоморфизм кольца многочленов Лорана $P\left[x^{ \pm 1}, c^{ \pm 1}\right]$, при котором $f(x, c) \mapsto f(-1, c)$ для любого $f(x, c) \in P\left[x^{ \pm 1}, c^{ \pm 1}\right]$. Получим,

$$
f_{0}(-1, c)(2-c)=1 \text {. }
$$

Но элемент $2-c$ необратим в $P[N]$. Теорема доказана.

ЗАмечАниЕ. Автор благодарен рецензенту, который сообщил, что утверждение первой теоремы верно для $\Omega_{2}$-групп. Точнее, группа $G$ называется $\Omega_{2}$-группой, если для любых двух конечных множеств $A, B \subset G$, каждое из которых состоит не менее чем из двух элементов, среди элементов $a b(a \in A, b \in B)$, имеется хотя бы два элемента, не равные остальным элементам вида $a b$.

В [7] доказано предложение 2.4, из которого следует, что для произвольного поля $P$ и произвольной $\Omega_{2}$-группы $G$ групповая алгебра $P[G]$ имеет только тривиальные единицы. Поэтому утверждение теоремы 1 справедливо для произвольного поля и любой неабелевой $\Omega_{2}$-группы.

\section{СПИСОК ЦИТИРОВАННОЙ ЛИТЕРАТУРЫ}

[1] I. Kaplansky, Fields and Rings, The Univ. Chicago Press, Chicago, IL, 1969. [2] M. S. Montgomery, Bull. Amer. Math. Soc., 75:3 (1969), 539-540. [3] C. Polcino Milies, S.K. Sehgal, An Introduction to Group Rings, Algebr. Appl., 1, Kluwer Acad. Publ., Dordrecht, 2002. [4] Х. Басс, Алгебраическая K-теория, Мир, М., 1973. [5] В. А. Артамонов, А. А. Бовди, Итоги науки и техн. Сер. Алгебра. Топол. Геом., 27, ВИНИТИ, М., 1989, 3-43. [6] D. Passman, The Algebraic Structure of Group Rings, Pure Appl. Math., John Wiley \& Sons, New-York, 1977. [7] А. Е. Залесский, А. В. Михалёв, Итоги науки и техн. Сер. Соврем. пробл. мат., 2, ВИНИТИ, М., 1973, 5-118.

\section{Е. И. Тимошенко}

Новосибирский государственный технический университет

E-mail: eitim45@gmail.com
Поступило

21.10 .2014

Исправленный вариант 02.02 .2015 